\title{
Empoderamiento y proactividad ante la Esclerosis Múltiple
}

\author{
Empowerment and proactivity against \\ multiple sclerosis
}

\section{Palabras clave}

Esclerosis Múltiple, empoderaramiento, proactividad, ciberactivismo.

\section{Keywords}

Multiple sclerosis, empowerment, proactivity, cyberactivism.

\section{Esclerosis Múltiple y autogestión de la enfermedad}

La Esclerosis Múltiple (EM) es una enfermedad crónica del sistema nervioso central. Se suele diagnosticar principalmente en adultos jóvenes. Puede producir una gran diversidad de síntomas, pero no se conoce ni su causa ni su curación. En nuestro país, 46.000 personas tienen EM y cada 5 horas se diagnostica un nuevo caso.

Para lograr una autogestión eficaz de una enfermedad, es fundamental que la persona afectada asuma el reto de 'trabajar por su salud' desde los aspectos emocional, médico y social. Esclerosis Múltiple España (<http://www.esclerosismultiple.com>) destaca, en su plan estratégico, la promoción de la salud y la calidad de vida de las personas con Esclerosis Múltiple (EM) hasta encontrar una solución definitiva a esta enfermedad, y aboga por el empoderamiento de la persona con EM como la mejor manera para conseguir mejoras tanto individuales como colectivas.

El Dr. Alejandro Jadad, innovador $\mathrm{y}$ fundador del Centre for Global eHealth Innovation de la Universidad de Toronto (Canadá), desafió a la Organización Mundial de la Salud

\section{Esclerosis Múltiple \\ España \\ <info@esclerosismultiple.com>}

Para citar:

Esclerosis Múltiple España (20I3): "Empoderamiento y proactividad ante la Esclerosis Múltiple", Revista Española de Discapacidad, I (I): 225-23I.

doi: <http://dx.doi.org/IO. $5569 / 2340-$ 5IO4.OI.OI.I2> 
La esclerosis múltiple es muy heterogénea en sus manifestaciones, por eso se suele llamar 'la enfermedad de las mil caras'.

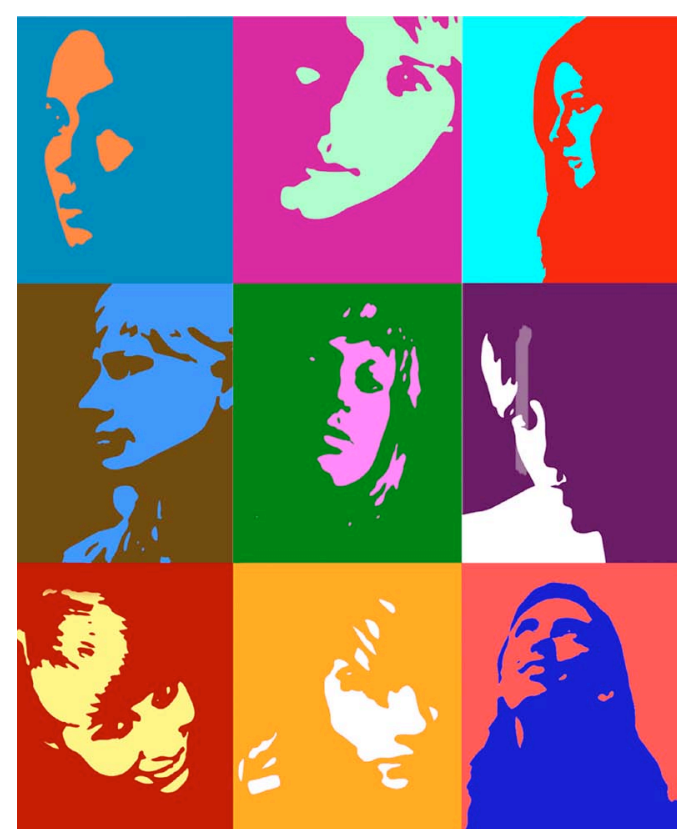

(OMS) al plantearle, en 2008, la necesidad de redefinir el término de salud, entendido desde I948 como "el estado de completo bienestar físico, mental y social, y no solamente la ausencia de enfermedad" (OMS, 2006:I). Jadad, junto otros expertos en salud, propuso un nuevo e interesante concepto de salud, definiéndola como la "capacidad que tiene un individuo o una comunidad para adaptarse y gestionar su vida al enfrentar desafíos físicos, mentales y sociales" (Docor Comunicación, 2013).

Esta capacidad de adaptación condiciona muy especialmente el bienestar de los afectados de Esclerosis Múltiple, ya que se trata de una enfermedad impredecible, al manifestarse mediante brotes, de forma aleatoria y caprichosa, los cuales pueden suponer un giro radical en la vida de las personas afectadas. Por esto, la EM exige a estas personas la adopción de estrategias que les aporten la flexibilidad necesaria para afrontar estos cambios o modificaciones inesperadas del transcurso de la enfermedad.

Para ello, resulta de gran utilidad una planificación vital que permita tomar decisiones informadas, que -por las características de la EM- sea flexible en su planteamiento, que promueva la salud y el autocuidado, y que favorezca en todos los aspectos posibles la autonomía personal y la participación en la comunidad.

Diversos estudios demuestran que cuanto más involucrados están los pacientes en su propia salud, más cuidado y atención ponen en la relación con los profesionales que les atienden, mejores decisiones toman y mejores resultados obtienen. La persona con Esclerosis Múltiple no es una excepción. Su implicación y la de su familia en la toma de decisiones relativas a la enfermedad se traduce en una mejor autogestión de ésta, que supone adquirir la confianza necesaria para poder gestionar de forma eficiente los aspectos emocionales, médicos y sociales ligados a la enfermedad.

En este aspecto, la búsqueda constante de recursos e información de los pacientes en pos de estar más capacitados para participar en las decisiones que afectan a su salud, sumada a la evolución de las tecnologías digitales, han supuesto el nacimiento de un nuevo tipo de paciente: el e-paciente. El e-paciente es un paciente proactivo, experto usuario de Internet, dinámico en la generación e intercambio de información sobre su enfermedad, implicado en el mantenimiento de su calidad de vida e interesado en contribuir al tratamiento y a la investigación relacionada con la salud y la mejora del sistema sociosanitario. Por otra parte, participa activamente en la generación de conocimiento, es artífice de la modificación de la relación médico-paciente y parte esencial del movimiento de las comunidades de pacientes.

En el ámbito que nos ocupa, las organizaciones deben dar respuesta, pues, a un perfil diferente de persona con Esclerosis Múltiple, con nuevas necesidades e inquietudes, y que se relaciona frecuentemente por Internet. Hemos de ofrecerle nuevas herramientas que favorezcan el empoderamiento de los afectados y la proactividad, para una adecuada autogestión de la enfermedad. Ambos conceptos, que están muy relacionados entre sí, resultan claves a la hora de convivir con enfermedades como la Esclerosis Múltiple. 
La proactividad es la actitud en la que el sujeto asume el pleno control de su conducta vital de modo activo, lo que implica tomar la iniciativa en el desarrollo de acciones creativas y audaces para generar mejoras, haciendo prevalecer la libertad de elección sobre las circunstancias de la vida. No significa sólo tomar la iniciativa, sino asumir la responsabilidad de hacer que las cosas sucedan; decidir en cada momento lo que queremos hacer y cómo lo vamos a hacer.

\section{El empoderamiento para la salud}

es un proceso mediante el cual las personas adquieren un mayor control sobre las decisiones y acciones que afectan a su salud [...]. Se establece una distinción entre el empoderamiento para la salud del individuo y el de la comunidad. El empoderamiento para la salud individual se refiere principalmente a la capacidad del individuo para tomar decisiones y ejercer control sobre su vida personal. El empoderamiento para la salud de la comunidad supone que los individuos actúen colectivamente con el fin de conseguir una mayor influencia y control sobre los determinantes de la salud y la calidad de vida de su comunidad, siendo éste un importante objetivo de la acción comunitaria para la salud (OMS, I998: I6).

\section{La información fiable es el mejor punto de partida}

Prepararse para tomar las decisiones más adecuadas en materia de salud casi siempre implica un acceso a información completa y fiable sobre la enfermedad. Esta búsqueda de información se intensifica cuando se trata de una enfermedad crónica -como es el caso de la Esclerosis Múltiple-, casi siempre con el fin de reducir la incertidumbre, aumentar el control de los síntomas y prevenir posibles efectos. Internet es, actualmente, el medio más utilizado para acceder e intercambiar información.

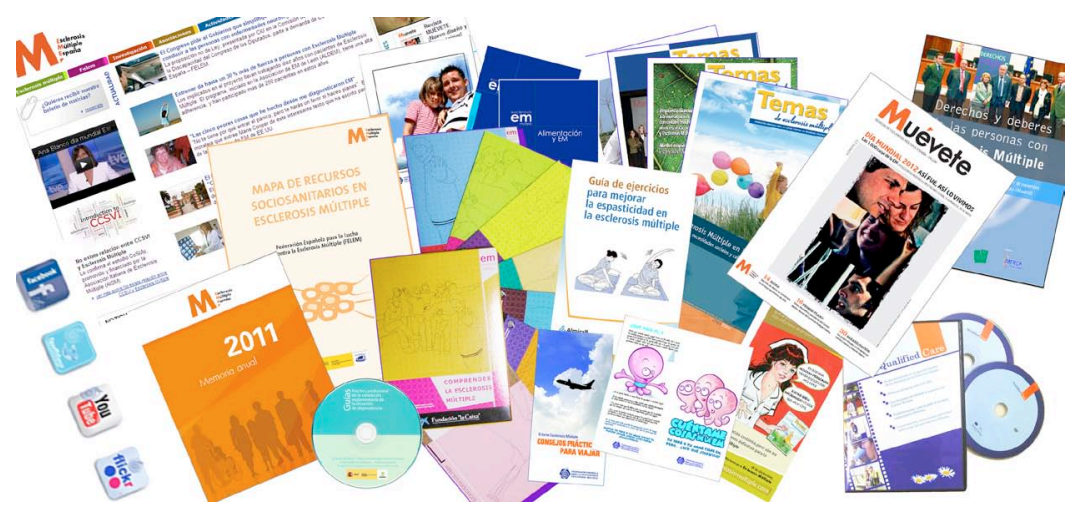

Los medios sociales sirven a las personas con Esclerosis Múltiple para intercambiar información sobre el diagnóstico, el tratamiento, la investigación y los avances, así como otros aspectos importantes de la enfermedad y su desarrollo. Estos medios facilitan que las personas afectadas se interrelacionen y compartan experiencias, lo cual supone un importante apoyo emocional, de utilidad terapéutica, así como que los profesionales se nutran de esta valiosísima información volcada por las personas con EM.

Pero estas herramientas van más allá. Además de servir a personas afectadas y profesionales para obtener los medios necesarios para comprender y trabajar con la enfermedad, la Web 2.0 hace posible la existencia del ciberactivismo, una nueva forma de movilización y promoción de causas que implica la participación directa y activa de los afectados en la mejora de su calidad de vida y la búsqueda de una solución a la enfermedad.

Así, resulta imprescindible el trabajo en red en la búsqueda de la transformación social. Trabajar en red requiere el empoderamiento de las organizaciones y personas que conforman esas redes, fortaleciéndolas como grupo social, para que puedan provocar cambios positivos en su contexto. El compromiso de las organizaciones de personas con Esclerosis Múltiple pasa por informar y educar en salud, de manera que puedan acceder a la información fiable, comprenderla y utilizarla para mantener y promover una buena calidad de vida. 


\section{El apoyo de los profesionales de la salud}

La atención, la formación y el asesoramiento a la persona por parte de profesionales de la salud expertos en la Esclerosis Múltiple es imprescindible en la autogestión adecuada de la enfermedad. La formación del paciente en educación sanitaria, complementada con aspectos relacionados con hábitos de vida saludable y estrategias de autocuidado, favorece una mayor adherencia a los tratamientos médicos y permite instaurar mecanismos de prevención ante posibles consecuencias adversas de la enfermedad. Así, con el apoyo de los profesionales, la persona con Esclerosis Múltiple se convierte en un mejor "trabajador de su propia salud".

El abordaje profesional ante personas afectadas de Esclerosis Múltiple se inicia con el diagnóstico. Desde ese mismo momento, el tratamiento debe enfocarse de manera integral, considerando todas las necesidades del paciente y de su entorno familiar, centrándolo en la persona y no en la enfermedad, e implicando a la familia en la atención y el apoyo necesarios en cada caso. Se recomienda el uso de canales informativos de calidad que reúnan información clínica y sociosanitaria, lo mismo que el desarrollo de de la atención y rehabilitación de organizaciones de esclerosis múltiple.

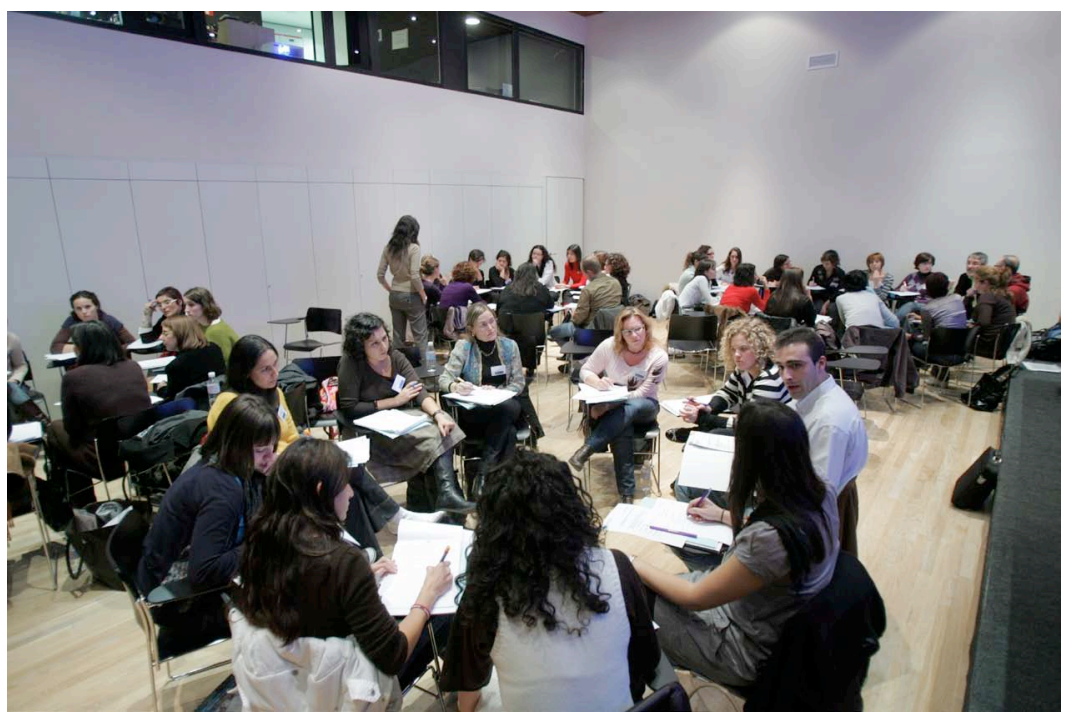

planes de cuidados personalizados elaborados con el paciente, que incluyan el soporte psicológico a la persona afectada y a su entorno familiar para hacer frente al diagnóstico y favorecer la adaptación personal a vivir con la enfermedad.

En muchos casos, recurrir a unos servicios de rehabilitación especializada y personalizada es la mejor opción para mantenerse lo más saludable posible, muy especialmente cuando las pautas que ofrecen los profesionales se extrapolan a la vida cotidiana.

\section{Vida activa: promoviendo la salud y la autonomía}

La Esclerosis Múltiple es una enfermedad neurodegenerativa. Por este motivo, mantener unos hábitos de vida saludables es imprescindible. La EM no permite en todos los casos un nivel intenso de actividad, dada la distinta forma en que afecta. Por ello, cada persona tiene que 'encontrar su punto', en armonía con su estilo de vida, que le permita desarrollar al máximo su capacidad mental y física. Mantener una vida lo más activa, autónoma y saludable posible es, sin excepción, clave para un abordaje adecuado de la EM en todo el proceso.

Además de la atención profesional mencionada anteriormente, es necesario integrar en la vida cotidiana otro tipo de hábitos. Practicar un deporte y realizar actividades en la naturaleza tienen un efecto tremendamente positivo en el colectivo de personas con EM. Dedicar tiempo a las aficiones y hobbies es también un estímulo para mantenerse activo, ilusionado y motivado, y ayuda a tener una buena autoestima. Otro aspecto de interés al hablar sobre vida activa es el voluntariado. La autogestión de una enfermedad puede beneficiarse enormemente tomando parte en el movimiento asociativo. 


\section{Activando los derechos}

Las asociaciones de pacientes también trabajan para que exista una mayor conciencia de la reivindicación y la defensa de derechos en el ámbito de la Esclerosis Múltiple. Para ello, es preciso tener un amplio conocimiento de las necesidades del colectivo que se representa, conocer cómo se articula el sistema sanitario, mantener una interlocución fluida con los organismos públicos correspondientes para desarrollar planes de acción y trabajar conjuntamente con otras organizaciones del sector.

La defensa de derechos de las personas con EM, representadas por las organizaciones de pacientes, organizaciones y profesionales del sector, puede ser más efectiva y gozar de más repercusión y apoyo si se implican en ellas las personas con EM, y cada vez existen más fórmulas que lo permiten. Esclerosis Múltiple España mantiene una comunicación continua con personas con EM, organizaciones y profesionales del sector, solicitando un feedback que permita establecer qué asuntos son prioritarios y requieren llevar a cabo un plan de actuación para defender los derechos de las personas con Esclerosis Múltiple.

\section{Iniciativas de Esclerosis Múltiple España relacionadas con el empoderamiento}

Algunas de las iniciativas más interesantes de Esclerosis Múltiple España -y las organizaciones integradas en esta plataforma- relacionadas con el empoderamiento y la proactividad son las siguientes:

- Esclerosis Múltiple España y sus entidades miembros ofrecen información a las personas interesadas en la enfermedad a través de diferentes canales: boletines electrónicos que recogen las noticias

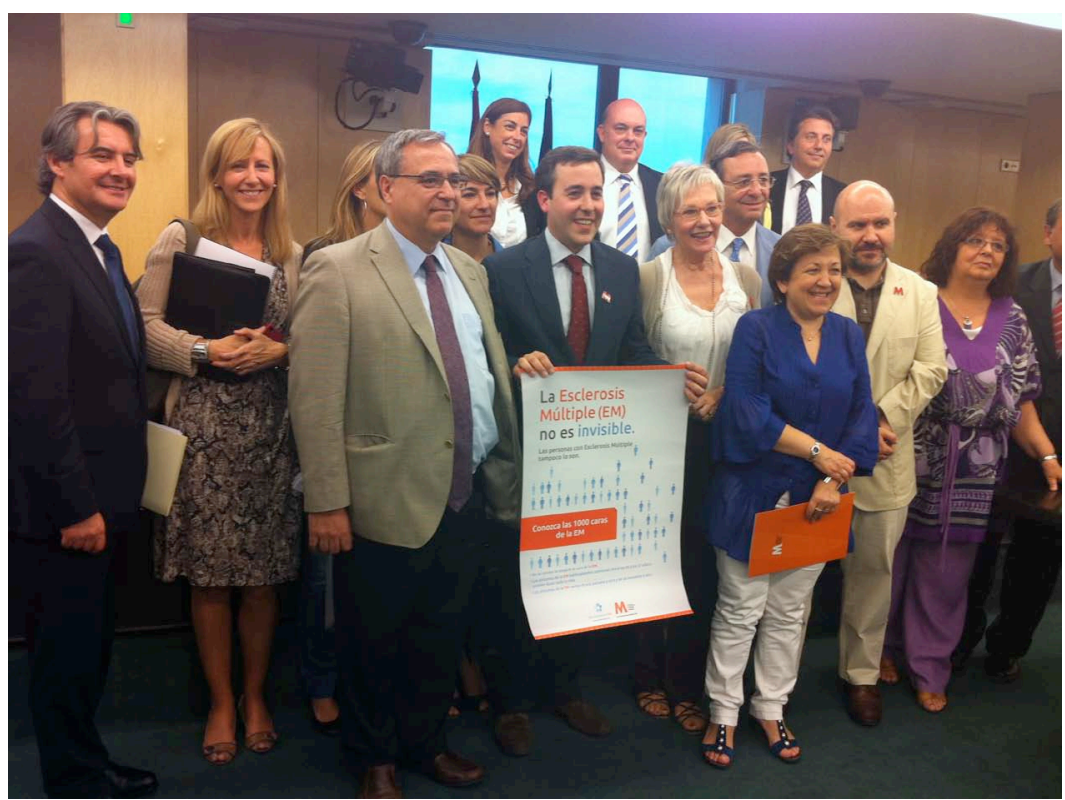

Representantes de Esclerosis Múltiple España y la Sociedad Española de Neurología, en el Congreso de los Diputados el Día Mundial de la Esclerosis Múltiple.
Muévete, los medios sociales de Internet, publicaciones y trabajos en colaboración con instituciones y entidades del sector, jornadas, conferencias, seminarios y talleres de formación.

- En las organizaciones de EM se desarrollan un gran número de iniciativas y campañas de sensibilización que fusionan deporte y solidaridad, como 'Mójate por la Esclerosis Múltiple’, iniciada en I 994 por la Fundación Esclerosis Múltiple y coordinada por Esclerosis Múltiple España.

- Las organizaciones de personas con Esclerosis Múltiple facilitan a las personas afectadas servicios personalizados y especializados de rehabilitación integral multidisciplinar (atención psicológica/ neuropsicológica, trabajo social, fisioterapia, terapia ocupacional, ocio, enfermería, logopedia o yoga, entre otras).

- Esclerosis Múltiple España promueve encuentros entre los profesionales de sus entidades miembros para el intercambio de experiencias, la unificación de criterios y la actualización de conocimientos. En 


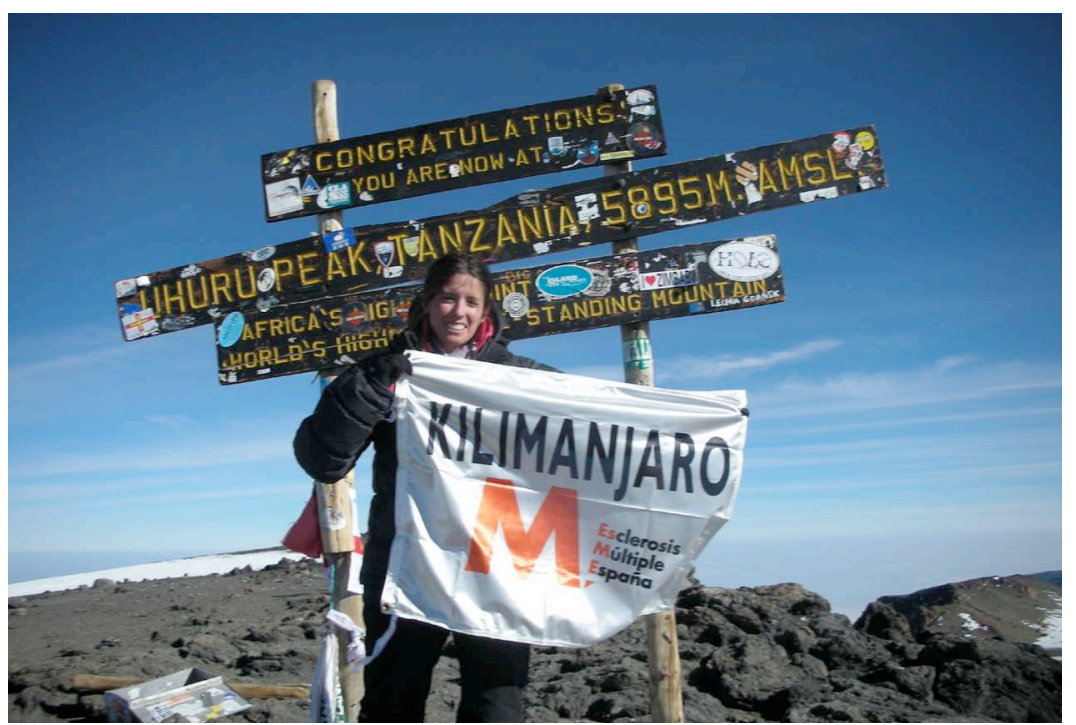

Una joven con esclerosis

múltiple participó en el ascenso al Kilimanjaro de un grupo de personas con esclerosis múltiple y párkinson en $20 \mathrm{II}$, gracias a la iniciativa 'Empoderamiento a través de la Aventura', apoyada por Esclerosis Múltiple España. esta línea, impulsa la elaboración de publicaciones sobre los síntomas más comunes, en colaboración con profesionales de sus entidades miembros expertos en la materia.

- Las organizaciones de pacientes y personas afectadas por Esclerosis Múltiple cuentan, desde diciembre de 20 I I, con un mapa de recursos sociosanitarios, fruto de la colaboración entre el Real Patronato sobre Discapacidad y Esclerosis Múltiple España. El Mapa de recursos sociosanitarios en Esclerosis Múltiple es de enorme utilidad para las asociaciones del ámbito social, profesionales, así como para las personas con EM y sus familias, pues permite conocer con mayor precisión los recursos y dispositivos de información disponibles sobre la enfermedad a escala estatal e internacional, al tiempo que pretende fomentar vías de colaboración de trabajo en red entre agentes activos en la promoción y mejora de la calidad de vida del colectivo.

- El programa 'Jóvenes con Esclerosis Múltiple y Vida Activa', desarrollado en colaboración con el Real Patronato sobre Discapacidad, se aproxima al movimiento de jóvenes con EM y recoge aquellas cuestiones que parecen importantes para este colectivo, con el fin de detectar estrategias para una autogestión eficaz de la enfermedad y el abordaje desde el sector asociativo. Para ello, Esclerosis Múltiple España promueve encuentros sobre EM y vida activa con responsables de organizaciones y con personas con la enfermedad. Las redes sociales también son un recurso de gran interés para conocer de primera mano las inquietudes de las personas afectadas por la enfermedad.

- En la línea estratégica de defensa de derechos de las personas con EM, Esclerosis Múltiple España lleva a cabo actuaciones de reivindicación y acción política para favorecer modificaciones normativas que aseguren los intereses y derechos de las personas con EM, y se adapten a su realidad y necesidades en los distintos ámbitos. En este sentido, Esclerosis Múltiple España está desarrollando una particular incidencia en la consecución de cambios normativos en varias líneas de trabajo:

- Acceso a medicamentos y tecnologías sanitarias.

- Reconocimiento del $33 \%$ de discapacidad con el diagnóstico de la enfermedad.

- Cuestiones relacionadas con el carné de conducir.

- La federación ha publicado una Guía jurídica para la defensa de los derechos de las personas con Esclerosis Múltiple, cuyo objetivo es ayudar a las personas con esta enfermedad a superar dudas o temores a la hora de reclamar sus derechos, y facilitar un mayor conocimiento de éstos, así como de las posibles actuaciones y cauces generales para ejercerlos.

- Para lograr de manera eficiente y eficaz sus objetivos, Esclerosis Múltiple España -en consonancia con entidades de EM referentes en el marco europeo y mundial en la promoción de la vida saludable y activa- une sinergias con entidades de EM nacionales e internacionales, organizaciones de personas 
con enfermedades neurodegenerativas, instituciones públicas y políticas, empresas privadas, organizaciones que representan a profesionales vinculados a la atención de personas con EM y entidades de la salud, discapacidad y solidaridad.

Las entidades de personas con EM realizan una importantísima labor en la atención a las personas afectadas. Es fundamental que cuenten con apoyos para que los servicios que ofrecen gocen de una calidad cada vez mayor y el movimiento asociativo se fortalezca.

Esclerosis Múltiple España agradece el apoyo del Real Patronato sobre Discapacidad en el desarrollo de investigaciones de carácter sociosanitario, que en los últimos años han permitido a la entidad conocer en mayor profundidad la situación de las personas con EM en España y difundir esa información.

Referencias bibliográficas

Docor Comunicación (2013): Alex Jadad: 'La salud es la capacidad de adaptación ante los desafíos' [nota de prensa], Bilbao: Docor Comunicación (en línea), <http://bit.ly/I6Ke99L>, acceso I de noviembre de 2012.

Esclerosis Múltiple España (20I2): Jóvenes con Esclerosis Múltiple y vida activa, Madrid: Real Patronato sobre Discapacidad (en línea), <http://www.esclerosismultiple.com/ publicaciones/Jovenes \% 2ocon \% 20EM\% 20 y \% 2ovida\% 2oactiva.pdf $>$, acceso I I de enero de 2013 .

- (20I I): Mapa de recursos sociosanitarios en Esclerosis Múltiple, Madrid: Real Patronato sobre Discapacidad (en línea), <http://www. esclerosismultiple.com/documentos/noticias/
MapaRecursosFELEM\% 2odefinitivo.pdf>, acceso I de noviembre de $20 \mathrm{I} 2$.

OMS (2006): "Constitución de la Organización Mundial de la Salud”, en Organización Mundial de la Salud: documentos básicos, $45^{\text {a }}$ edición (en línea), <http://www.who.int/ governance/eb/who_constitution_sp.pdf>, acceso I de noviembre de $20 \mathrm{I} 2$.

- (I998): Glosario de promoción de la salud. Madrid, Ministerio de Sanidad y Consumo (en línea), <http://www.msc.es/profesionales/ saludPublica/prevPromocion/docs/glosario. pdf $>$, acceso I de noviembre de $20 \mathrm{I} 2$.

Traver, V. y Fernández-Luque, L. (coords.) (20I I): El ePaciente y las redes sociales, Fundación Vodafone; ITACA. 